\title{
LncRNA H19 from T-Regulatory-Cell-Derived Exosomes Sponges miR-154-5p to Promote Osteogenic Differentiation of BMMSCs Through Upregulating POSTN
}

\section{Zhang Jing ( $\nabla_{\text {jingz77@163.com ) }}$}

Xuzhou Central Hospital, Xuzhou Clinical College of Xuzhou Medical University

\section{Zhigang Li}

Xuzhou Medical University

Cuicui Liu

Xuzhou Medical University

Yaxing Wu

Xuzhou Medical University

Chenchen Li

Xuzhou Medical University

Meng Jian

Xuzhou Medical University

\section{Research Article}

Keywords: LncRNA, T-Regulatory-Cell-Derived exosomes, Bone marrow mesenchymal stem cells, MiR-154$5 p$, Osteogenic differentiation, POSTN

Posted Date: February 11th, 2021

DOl: https://doi.org/10.21203/rs.3.rs-175823/v1

License: (a) (i) This work is licensed under a Creative Commons Attribution 4.0 International License. Read Full License 


\section{Abstract}

Background: Mesenchymal stem cells (MSCs) can play an important role in anti-inflammatory function because it can induce Treg cells formation. Recent studies have confirmed that Tregs can release exosomes and induce immune tolerance as important immune cells. Thus, to clarify the function and regulatory mechanism of Treg cells-derived exosomes on osteogenic differentiation of BMMSCs is very important.

Methods: CD4+ T cells isolated from mouse spleens were induced into CD4+ Foxp3+ Treg cells and cocultured with BMMSCs. Then we isolated exosomes derived from Treg cells and tracked the exosomes using fluorescent labeling PKH67.

Starbase, miRWalk, targetscan, bioinformatics analysis and functional complementation experiments to find relative IncRNA-miRNAs which involed in osteogenic differentiation of BMMSCs.

Results: We found that exosomes derived from Tregs could enter BMMSCs and the expression of osteoblast-related genes were significantly up-regulated compared to the BMMSCs co-cultured with naïve T cells. LncRNA H19 from Tregs-derived exosomes could affect the osteogenic differentiation of BMMSCs in vitro by regulating POSTN expression via sponge miR-154-5p.

Conclusions: This study demonstrated that important role of exosomes from Tregs in regulating osteogenic differentiation of BMMSCs. IncRNA H19 from Tregs-derived exosomes plays a positive regulatory role in osteogenic differentiation of BMMSCs through miR-154-5p/POSTN axis. It might has important clinical implications for inflammatory diseases and exosomes released from Tregs could be used as a new type of immunoregulation reagent for the treatment of periodontitis.

\section{Introduction}

Periodontitis is a chronic infectious disease that leads to a progressive destruction of periodontal tissue and is a major cause of tooth loss in adults[1]. Though conventional therapies can successfully control periodontal inflammation but often cannot achieve recovery of damaged periodontal tissue. In recent decades, the biomedical applications of mesenchymal stem cells (MSCs) have attracted increasing attention and various stem cells have been investigated for periodontal regeneration, including periodontal ligament stem cells (PDLSCs), bone marrow mesenchymal stem cells (BMMSCs), induced pluripotent stem cells and et al [2-5]. Many studies have demonstrated that human PDLSCs has the potential for use as a practical cell-based treatment for periodontal diseases[6, 7]. However, access to the periodontal ligament requires removal of teeth and the number of recoverable PDLSCs is limited due to their rarity and the small sample size. In contrast, BMMSCs are being increasingly used for periodontal regeneration because they can be harvested in much larger numbers and relative ease of acquisition[8, 9]. Previous studies also revealed that periodontal ligament stem cells had impaired immunomodulatory function after exposure to an inflammatory environment[10]. Recently, some results showed that BMMSCs had higher osteogenic capacity than PDLSCs when used the PDLSCs and BMMSCs with 
biomaterials to repair bone defects in beagle alveolar bone[11]. Our preliminary study also showed that BMMSCs had higher osteogenic capacity than PDLSCs under the inflammatory conditions. We speculated that the BMMSCs might own the stronger immunomodulation in local microenvironment via anti-inflammatory functions, compared to PDLSCs[12].

In recent years, the regulatory effect of mesenchymal stem cells (MSCs) on immune cells has drawn more and more attention. Studies have demonstrated that when exposed to an inflammatory environment, MSCs can orchestrate local and systemic innate and adaptive immune responses through the release of various mediators, including immunosuppressive molecules, growth factors, exosomes, chemokines, complement components and various metabolites[13-16]. As the central link of immune regulation, the role of $\mathrm{T}$ cells in periodontal inflammatory alveolar bone absorption has been confirmed by more and more studies [17]. Among them, regulatory T cells (Tregs) are important immunoregulatory functional cells. Treg cells prevent inflammatory damage but the precise mechanisms of suppression are incompletely understood[18-21]. Recent studies have confirmed that MSCs can secrete soluble factors, including TGF- $\beta 1$, IL-6, IL-8, IDO, PGE2 and so on. These soluble factors make MSCs interact with immune cells and exert their anti-inflammatory and immunosuppressive effects by regulating the local microenvironment of injury and reducing the secretion of inflammatory factors [22, 23]. Therefore, MSCs can play an important role in anti-inflammatory function because it can induce Treg cell formation [24, 25].

Previous studies have found that Tregs-mediated anti-inflammatory microenvironment promotes bone regeneration. For example, overexpression of the Foxp3 gene in mice can protect bone loss from ovariectomy [26]. CD4 ${ }^{+}$Foxp $^{+}$Tregs enhance MSCs-mediated bone formation by inhibiting the number of neutrophil infiltration and the expression of IFN-y, IL-6 and TNF- $\alpha$ in the MSCs receptor implantation area [27]. However, how MSCs (including BMMSCs) interact with Treg cells is not clear. Therefore, studying the regulatory mechanism of the interaction between BMMSCs and Tregs is of great value for the repair of periodontal bone defects. Recently, a large number of studies have shown that IncRNAs and miRNAs act as ceRNAs to inhibit each other, forming an accurate regulatory network and regulating the target genes of miRNAs. For example, LncRNA LINC00707 sponges miR-370-3p to promote osteogenesis of human BMMSCs through up-regulating WNT2B [28]. LncRNA MALAT1 promotes osterix expression to regulate osteogenic differentiation by targeting miRNA-143 in human BMMSCs [29]. Although roles of IncRNA-miRNA networks in BMMSCs osteogenic differentiation have been reported, their functions in the regulatory mechanism of the interaction between BMMSCs and Tregs remain poorly understood.

In this study, we found that coculture of Tregs and BMMSCs can promote osteogenic differentiation of BMMSCs. To further clarify the regulatory mechanism, we isolated and purified exosomes which are released from Tregs. The results showed that IncRNA H19 from tregs-derived exosomes can be bound by miR-154-5p, and thus regulate the expression of its target gene periostin (POSTN). In summary, our study demonstrates that the H19-miR-154-5p-POSTN signal axis plays a key role in the regulation of osteogenic differentiation of BMMSCs. 


\section{Materials And Methods \\ Cell culture}

Human bone marrow-derived mesenchymal stem cells (BMMSCs) were purchased from ScienCell, (CA, USA, catalog number:7500) and maintained in mesenchymal stem cell medium, consisting of basal medium, $5 \%$ of fetal bovine stem serum, $1 \%$ mesenchymal stem cell growth supplement and penicillin/streptomycin solution (MSCM, ScienCell, catalog number:7501). The passages from 5 th to 8 th were used in all the experiments.

\section{$\mathrm{CD4}^{+}$naïve T cells isolation and in vitro induction of Treg cells differentiation}

The splenocytes were washed with PBS for 3 times and the mouse naïve $\mathrm{CD} 4^{+} \mathrm{T}$ cell isolation kit was used to isolate the naïve $\mathrm{CD} 4^{+} \mathrm{T}$ cells according to the manufacturer's instruction. In Brief, CD $4^{+}$ activated/memory $\mathrm{T}$ cells and non-CD4 ${ }^{+} \mathrm{T}$ cells were depleted by indirect magnetic labeling using a cocktail of biotin-conjugated antibodies against various markers including CD8a, CD11b, CD11c, CD19, CD25, CD45R, CD49b, CD105, anti-MHC class II, Ter-119 and TCR $\gamma / \delta$ followed by addition of antibiotin microbeads. $5 \mu \mathrm{g} / \mathrm{ml}$ plate-bound anti-CD3, $2 \mu \mathrm{g} / \mathrm{ml}$ anti-CD28, $2 \mathrm{ng} / \mathrm{ml} \mathrm{TGF} \beta$ and $100 \mathrm{Unit} / \mathrm{ml} \mathrm{IL-2}$ were used to activate the isolated cells $\left(1 \times 10^{6}\right.$ cells/well). The cells were stained with FITC-conjugated antiCD45RA and APC-conjugated anti-CD4 antibodies. After washing, the cells were fixed and made permeable using permeabilization buffer and then stained with PE-labeled anti-Foxp3 antibody. The tests were detected by flow cytometry.

\section{RNA extraction and qRT-PCR}

The extraction of total RNA and the analysis of qRT-PCR were performed according to the previous description [30]. We used TRIZOL reagent (Thermofisher, USA) to extract total RNA by in cells and tissues. Taqman probes (Applied Biosystems, USA) were used to quantify miRNAs. Briefly, $1 \mu \mathrm{g}$ of total RNA was transcribed to CDNA using AMV reverse transcriptase (Takara, Japan) and a RT primer. The reaction conditions were: $16^{\circ} \mathrm{C}$ for $30 \mathrm{~min}, 42^{\circ} \mathrm{C}$ for $30 \mathrm{~min}$ and $85^{\circ} \mathrm{C}$ for $5 \mathrm{~min}$. Real-time PCR was performed using a Taqman PCR kit on an Applied Biosystems 7300 sequence detection system (Applied Biosystems, USA). The reactions were performed in a 96 -well plate at $95^{\circ} \mathrm{C}$ for $10 \mathrm{~min}$, followed by 40 cycles of $95^{\circ} \mathrm{C}$ for $10 \mathrm{sec}$ and $60^{\circ} \mathrm{C}$ for $1 \mathrm{~min}$. U6 was used as the internal control.

\section{Western blotting analysis}

Cells were lysed in 1\% n-octyl-p-D-glucopyranoside (OG) buffer (20 mM Tris- $\mathrm{HCl}(\mathrm{pH} 8.0), 150 \mathrm{mM} \mathrm{NaCl}$, 1\% OG, $1 \mathrm{mM}$ EDTA, $10 \mathrm{~g} / \mathrm{ml}$ leupeptin, $2 \mathrm{~g} / \mathrm{ml}$ aprotinin, $1 \mathrm{mM}$ PMSF). The total protein density was determined using bicinchoninic acid (BCA) protein assay kit (synthgene, China). The protein was then incubated overnight with the following primary antibodies at $4^{\circ} \mathrm{C}$ : CD9, CD63 and TSG101 (1:500, SBI, USA), POSTN (1:1500, Abcam, USA), GAPDH (1:2000, Abcam, USA). GAPDH served as a loading control. 
After incubation with the corresponding second antibodies, protein bands were quantified using Image $\mathrm{J}$ Software.

\section{In vitro mineralization assay}

Mesenchymal stem cell osteogenic differentiation medium (MODM, Sciencell, Sandiego, CA, USA) was used to induce osteogenic differentiation of BMMSCs. After 3 days of induction, alkaline phosphatase (ALP) activity was assayed with an ALP activity kit according to the manufacturer's protocol (SigmaAldrich). To detect mineralization, cells were induced for 2 weeks, fixed with $70 \%$ ethanol and stained with $2 \%$ Alizarin Red (Sigma-Aldrich). To quantify the calcium mineral density, Alizarin Red was destained with $10 \%$ cetylpyridinium chloride (CPC) in $10 \mathrm{mmol} / \mathrm{L}$ sodium phosphate for 30 minutes at room temperature. The calcium concentrations were determined by an absorbance measurement at $562 \mathrm{~nm}$ on a multiplate reader and compared to a standard calcium curve with calcium dilutions in the same solution. The final calcium level in each group was normalized to the total protein concentration detected in a duplicate plate.

\section{Exosome isolation and labeling}

Exosome-depleted FBS was used in the following experiments to avoid the impact of exosomes. FBS was depleted of exosomes by ultracentrifugation at $1 \times 10^{6} \mathrm{~g}$ at $4^{\circ} \mathrm{C}$ for $16 \mathrm{~h}$ (Beckman Coulter Avanti J-30l, USA). After being incubated for 48-72 h, the culture medium was harvested and exosomes were isolated by ultracentrifugation. Briefly, cell culture medium was sequentially centrifuged at $300 \mathrm{~g}$ for $10 \mathrm{~min}, 2,000$ $\mathrm{g}$ for $15 \mathrm{~min}$, and $12,000 \mathrm{~g}$ for $30 \mathrm{~min}$ to remove floating cells and cellular debris. These were then passed through a $0.22-\mu \mathrm{m}$ filter. The supernatant was further ultracentrifuged at $1 \times 10^{6} \mathrm{~g}$ for $2 \mathrm{~h}$ at $4^{\circ} \mathrm{C}$, washed in phosphate-buffered saline (PBS), and submitted to a second ultracentrifugation in the same conditions. Exosomes were quantified with bicinchoninic acid (BCA) method. Exosomal protein was measured by BCA protein assay kit (synthgene, China). Purified exosomes were fluorescently labeled using PKH67 (Sigma, USA), according to the protocol.

\section{Transmission electron microscope}

Exosomes were precipitated and immediately fixed in $2.5 \%$ glutaraldehyde at $4^{\circ} \mathrm{C}$ for the electron microscope observation. After fixation, specimens were processed through dehydration in gradient alcohol, and infiltrated in epoxy resin and then embedded. The ultrathin sections were stained with uranyl acetate and lead citrate, and were observed under transmission electron microscope (TEM) (JEM-1010; JEOL,

Tokyo, Japan).

\section{NanoSight tracking analysis (NTA)}

Isolated exosomes were analyzed using Nanosight LM10 system (Nanosight Ltd, Navato, CA) equipped with a blue laser $(405 \mathrm{~nm})$. Nanoparticles were illuminated by the laser and their movement under 
Brownian motion was captured for 60 seconds and the video recorded was subjected to NTA using the Nanosight particle tracking software to calculate nanoparticle concentrations and size distribution.

\section{Exosome labeling}

For exosome tracking experiments, PKH67 membrane dye (Sigma) was used to label exosomes. Labeled exosomes were washed in $10 \mathrm{ml}$ of culture medium, collected by ultracentrifugation $\left(1 \times 10^{5} \mathrm{~g}, 2 \mathrm{~h}\right)$ and resuspended in culture medium. Exosome labeling with PKH67 (Sigma) was performed following the manufacturer's procedures.

\section{Luciferase reporter assay}

pMIR-POSTN-3'-UTR-WT (or pMIR-H19-3'-UTR-WT) as well as pMIR-POSTN-3'-UTR-MUT (or pMIRH19-3'-UTR-MUT) luciferase reporter plasmids were constructed by Synthgene Biotech (Nanjing, China). The sequences that could binding to miR-154-5p were partly mutated and inserted into the reporter plasmid in order to identify the binding specificity. The implementation method refers to previous study [31]. Briefly, BMMSCs cells were seeded in a 24 well plate until reaching $60 \%$ confluence. Each well was co-transfected with luciferase reporter plasmids $(0.5 \mu \mathrm{g})$ and RNA mimics $(100 \mathrm{pmol})$ using Lipofectamine 2000 (Thermofisher, USA) according to the manufacturer's protocol. The luciferase activity was measured after $48 \mathrm{~h}$ of transfection, by using the Dual-Luciferase Reporter Assay (Promega, Shanghai, China) according to the manufacturer's instructions and normalized to Renilla signals.

\section{RNA immunoprecipitation}

RIP assay was performed using an RNA Binding Protein Immunoprecipitation Kit (Millipore) according to the manufacturer's instructions with an anti-Ago 2 antibody ( $2 \mu \mathrm{g}$; Millipore) and normal mouse lgG as an NC. qPCR was performed using Taqman Universal PCR Mix as described above.

\section{RNA pull-down}

A Pierce ${ }^{\text {TM }}$ Magnetic RNA-Protein Pull-Down Kit (ThermoFisher, USA) was used to perform RNA pull-down assays according to the manufacturer's instruction. Biotinylated H19 RNA was synthesized by Synthgene (China). In brief, 50 pmol biotinylated RNA were incubated with $50 \mu$ l prewashed streptavidin-agarose beads for one hour at $4^{\circ} \mathrm{C}$ for each assay. Then, RNA-bound beads were incubated with lysates from BMMSC cells cytosolic/nuclear extracts and eluted proteins were detected by western blot.

\section{Statistical analyses}

All experiments were repeated three times and the data are presented as the mean \pm standard deviation using SPSS 18.0 (SPSS, inc.). One-way ANOVA and post hoc Dunnett's T3 test were performed in order to compare the differences among and between groups, respectively. $\mathrm{P}<0.05$ was considered to indicate a statistically signifcant result.

\section{Results}




\section{Tregs can promote osteogenic differentiation of BMMSCs in vitro}

To identify whether the Tregs has an effect on the differentiation of BMMSCs, we first isolated and identified mouse naïve CD4 $+\mathrm{T}$ cells of splenocytes. Then, we induced the differentiation of $\mathrm{CD} 4^{+} \mathrm{T}$ cells into $\mathrm{CD}^{+}{ }^{+}$Foxp $^{+}$Treg cells in vitro (Fig. 1A). We cocultured the naïve T cells with BMMSCs and Treg cells with BMMSCs respectively. The expression levels of Runx2, ALP and OCN in BMMSCs were determined. The results showed that the expression of osteoblast-related genes in the group of BMMSCs cocultured with Tregs was significantly up-regulated, whereas added exosome inhibitors (GW4869) resulted in decreased osteogenic gene expression (Fig. 1B). To further investigate the effects of Tregs on the osteogenic process of BMMSCs, ALP activity assay, calcium content assay, ALP staining, and alizarin red staining were performed and revealed that Tregs could promote osteogenic differentiation of BMMSCs (Fig. 1C-E). All these results suggest that Tregs could promote osteogenic differentiation of BMMSCs.

\section{Treg-Cell-Derived Exosomes can promote osteogenic differentiation of BMMSCs}

To determine whether Treg-Cell-Derived exosomes are involved in regulating osteogenic differentiation of BMMSCs, we isolated and identified exosomes from Tregs (Fig. 2A-C). To test whether Treg-Cell-Derived exosomes contributed to the osteogenic differentiation of BMMSCs in vitro, we tracked the exosomes using fluorescent labeling PKH67 (Fig. 2D). Based on this result, the mRNA expression of Runx2, ALP and OCN were tested. And ALP staining, alizarin red staining, ALP activity assay and calcium content assay in BMMSCs also were used to evaluate the osteogenic differentiation (Fig. 2E-G). All these results demonstrate that exosomes derived from Tregs are indeed involved in regulating osteogenic differentiation of BMMSCs.

\section{POSTN protein promotes osteogenic differentiation of BMMSCs}

The extracellular matrix (ECM) has recently been reported to play a vital role in bone formation and POSTN has been suggested as a key member in constructing the ECM in bone tissue [31]. To test the role of POSTN in the osteogenic differentiation, we used WB and PT-PCR(Fig. 3A-B). We found that POSTN overexpression markedly upregulated the mRNA levels of RUNX2, ALP and OCN. However, POSTN knockdown significantly downregulated the expression of RUNX2, ALP and OCN mRNA. To further investigate the effects of POSTN on the osteogenic process, ALP staining, alizarin red staining, ALP activity assay and calcium content assay were performed and revealed that POSTN overexpression increased ALP activity, calcium content and mineralized bone matrix formation in BMMSCs (Fig. 3C-E). Therefore, POSTN could promote osteogenic differentiation of BMMSCs. 


\section{MiR-154-5p affects osteogenic differentiation of BMMSCs by regulating the expression of POSTN.}

The expression levels of POSTN were determined with different concentration exosomes $(0,10,20$ and 30 $\mu \mathrm{L})$. And an obviously positive correlation between the expression levels of POSTN and exosomes concentration (Fig. 4A). However, the mRNA expression of POSTN was not significantly higher along with the increment of exosome concentration. (Fig. 4B). This data suggest that POSTN has a posttranscriptional regulatory mechanism in BMMSCs. Based on this prediction, we used Starbase, miRWalk and targetscan to find relative miRNAs and results showed that 26 miRNAs were screened (Fig. 4C). Five miRNAs (including miR-135b-5p, miR-154-5p, miR-296-3p, miR-185-5p and miR-18a-5p) were randomly selected, and the expression of these miRNAs with exosomal treated $(10 \mu \mathrm{L})$ BMMSCs was detected by qRT-PCR. The results showed that the expression of miR-154-5p was significantly downregulated (Fig. 4D). To further investigate the effects of miR-154-5p on the osteogenic process, RT-PCR was performed and revealed that there was an obviously negative correlation between the expression levels of miR-154-5p and Treg-Cell-Derived exosomes concentration (Fig. 4E). Following the above findings, luciferase reporter assay was used to confirm the binding site of miR-154-5p and POSTN (Fig. 4F). To further investigate whether miR-154-5p targets POSTN in BMMSCs, luciferase reporter constructs carrying a POSTN reporter were generated. Compared with control groups, POSTN-wt reporter activity was strongly inhibited by miR-154-5p mimic,While the POSTN-mut reporter was not affected by miR-154-5p (Fig. 4G). And a negative correlation between miR-154-5p and POSTN expression was confirmed (Fig. 4H). In addition, ALP staining, alizarin red staining, ALP activity assay and calcium content assay were also performed and revealed that miR-154-5p knockdown increased osteogenic differentiation of BMMSCs (Fig. 4I-K). Therefore, miR-154-5p could regulate osteogenic differentiation of BMMSCs.

\section{LINC H19 negatively regulates miR-154-5p}

It has previously been reported that the regulatory network (ceRNA) composed of IncRNAs and miRNAs plays an important role in the osteogenic differentiation of BMMSCs [32]. Through bioinformatics analysis (IncRNABase) and literature reading, 5 IncRNAs were found that are closely related to MSCs osteogenic differentiation, including IncRNA OG, IncRNA KCNQ10T1, IncRNA H19, IncRNA MEG3 and IncRNA MALAT1. The expressions of these IncRNAs after adding exosomes (Induced group) were detected by qRT-PCR. A heatmap describing the changes in IncRNAs is shown in (Fig. 5A). To further determine whether $\mathrm{H} 19$ regulates miR-154-5p, dual-luciferase reporter constructs carrying a $\mathrm{H} 19$ reporter were generated. The results showed that the $\mathrm{H} 19$-wt reporter activity was predominantly decreased in BMMSCs cells when transfected with miR-154-5p mimic, while the H19-mut reporter was not affected by miR-154-5p (Fig. 5B-C). In addition, the RNA immunoprecipitation (RIP) assay showed that compared with the anti-normal IgG group, $\mathrm{H} 19$ and miR-154-5p expression levels were significantly higher in the antiAgo2 group (Fig. 5D and E). RNA pull-down assays confirmed that $\mathrm{H} 19$ could directly bind with AGO2 in BMMSCs (Fig. 5F and G). To elucidate the key role of Treg-cell-derived exosomes in the H19-miR-154-5p signal axis, we co-incubated exosomes with BMMSCs and detected the expression of H19 using qRT-PCR. We found that Tregs-derived exosomes significantly increased the expression level of H19 in BMMSCs 
(Fig. $5 \mathrm{H}$ ). To determine whether $\mathrm{H} 19$ acts as an miRNA sponge that competes with mRNA for binding to miR-154-5p, we constructed H19-overexpressing, miR-154-5p-overexpressing, H19-knockdown and miR154-5p-knockdown BMMSCs cell lines, examined the expression of miR-154-5p and H19. The result showed that $\mathrm{H} 19$ has negative correlations with miR-154-5p (Fig. 5I-J). Taken together, our results provide strong evidence that $\mathrm{H} 19$ is able to directly bind to miR-154-5p and negatively regulate its expression.

\section{LncRNA H19 /miR-154-5p axis regulates POSTN expression during osteogenesis}

From previous research, $\mathrm{H} 19$ has negative correlations with miR-154-5p and POSTN is a direct target of miR-154-5p, so we assessed whether $\mathrm{H} 19$ could upregulate POSTN expression by sponging miR-154-5p. The expression levels of miR-154-5p were confirmed using functional complementation experiments. The results showed that $\mathrm{H} 19$ overexpression significantly decreased miR-154-5p expression, and cotransfection of miR-154-5p mimic rescued miR-154-5p expression (Fig. 6A). In contrast, H19 knockdown significantly increased miR-154-5p expression and co-transfection of miR-154-5p inhibitor decreased miR154-5p expression. The variation trend of POSTN protein was opposite to that of miR-154-5p, which was in line with expectations (Fig. 6B). Furthermore, the expression of Runx2, ALP and OCN were further confirmed at the miRNA level. Our results revealed that H19 overexpression significantly increased Runx2, ALP and OCN mRNA expression, while H19 knockdown significantly decreased Runx2, ALP and OCN mRNA expression, and co-transfection of miR-154-5p inhibitor rescued Runx2, ALP and OCN mRNA expression (Fig. $6 \mathrm{C}$ ). Then alkaline phosphatase (ALP) staining, alizarin red staining, ALP activity assay and calcium content assay were performed and revealed that $\mathrm{H} 19$ overexpression increased ALP activity and mineralized bone matrix formation in BMMSCs. In contrast, $\mathrm{H} 19$ knockdown significantly decreased ALP activity and mineralized bone matrix formation, and co-transfection of miR-154-5p inhibitor rescued ALP activity and mineralized bone matrix formation (Fig. 6D-F). In summary, the H19-miR-154-5p-POSTN axis regulated POSTN in BMMSCs during osteogenesis.

\section{LINC H19 regulates osteogenic differentiation of BMMSCs}

To further determine the effect of LncRNA H19 from Tregs-cell-derived exosomes on osteogenic differentiation of BMMSCs, we constructed H19-overexpressing and H19-knockdown Tregs cell lines and co-cultured with BMMSCs. H19 expression levels were confirmed using qRT-PCR (Fig. 7A). The results showed that Treg/H19-overexpressing group significantly increased the expression of $\mathrm{H} 19$ in BMMSCs and Treg/H19-knockdown group significantly reduced the expression of $\mathrm{H} 19$. To further investigate the effects of $\mathrm{H} 19$ on the osteogenic process, we assessed the mRNA expression levels of Runx2, ALP and OCN in BMMSCs (Fig. 7B). Next, we further evaluated the effect of H19 on BMMSCs osteogenic differentiation. $\mathrm{H} 19$ overexpression increased osteoid formation whereas knockdown of $\mathrm{H} 19$ resulted in decreased osteoid formation compared with the control group (Fig. 7C-E). Pearson's correlation scatter plots was used to further analyze the correlation between H19/miR-154-5p/POSTN (Supplementary Fig. 1). The results are consistent with expectations.

\section{Discussion}


Currently, it is well established that MSCs rely on different mechanisms to exert their suppressive properties under inflammatory microenviroments. One important mechanism is the capacity of MSCs to induce functional regulatory T cells (Tregs)[33-36]. Another study established that immune tolerance of human dental pulp-derived mesenchymal stem cells mediated by CD $4+$ CD $25+$ FoxP3 + Regulatory TCells and induced by TGF-beta1 and IL-10[37]. These studies confirmed that Tregs are a key population in immune tolerance and their potential use in the treatment of chronic inflammatory diseases has been increasingly investigated. Previous study found that Tregs affect osteogenic differentiation of MSCs in mouse models of postmenopausal osteoporosis[38]. Accordingly, herewith, we explored the effect of Treg cells on osteogenesis differentiation of BMMSCs. When coculture of Tregs and BMMSCs, we found that Tregs can promote osteogenic differentiation of BMMSCs. However the mechanisms of Treg cells in the regulation of osteogenesis differentiation of BMMSCs under inflammatory microenviroments has not been investigated.

A number of secretory molecules and transcription factors have been identified as regulators controlling osteoblastogenesis. It is well established that the exosomes are exciting newly recognized extracellular vesicles that transfer a variety of bioactive compounds[39-41]. Many cells can secrete exosomes, such as tumor cells, dendritic cells, CD 4 + and CD8 + T cells or cells from different tissues or organs[42-47]. Based on the constituents of exosomes, they are believed to transport intercellular information between tissue microenvironments. In this study, we isolated exosomes from Tregs and cocultured with BMMSCs, the results showed that exosomes could enters BMMSCs using fluorescent PKH67. And we found that the expression of osteoblast-related genes were significantly up-regulated compared to the BMMSCs cocultured with naïve T cells when exosomes cocultured with BMMSCs. This observation supports other studies revealing that exosomes from Tregs play a critical role in the immune regulation of MSCs and it can promote osteogenic differentiation of BMMSCs $[48,49]$.

POSTN is a $90-\mathrm{kD}$ a matricellular protein that is usually highly expressed in connective tissues such as periosteum, periodontal ligaments, and tendons[50]. Previously published studies have shown that POSTN plays an important role in bone formation and bone metabolism and can regulate bone homeostasis [51-53]. We also demonstrated that the mRNA levels of RUNX2, ALP, OCN and mineralized bone matrix formation were significantly increased when POSTN overexpression. We founded that there was a dose dependently between the expression levels of POSTN protein and Treg-cell-derived exosomes concentration. These observation supports that POSTN play a critical role in osteogenic differentiation of BMMSCs. While, the mRNA expression of POSTN was not significantly higher along with the increment of exosomes concentration. This result showed that POSTN has a post-transcriptional regulatory function.

MicroRNAs (miRNAs) emerge as important regulators of stem cell lineage commitment and bone development. Rencent study has shown that miR-154-5p expression was associated with metastasis of nasopharyngeal carcinoma[54].Other study confirmed that miR-154-5p functions as a tumor suppressor in glioblastoma[55]. Li et al. demonstrated that MiR-154-5p regulates osteogenic differentiation of adipose-derived mesenchymal stem cells under tensile stress through the Wnt/PCP pathway by targeting Wnt11 MSCs[56]. However, the role and potential molecular mechanism of miR-154-5p in osteogenic

Page 10/23 
differentiation of BMMSCs under inflammatory microenviroments is still undiscovered. In this study, we found the miR-154-5p expression was significantly decreased when Treg-cell-derived exosomes concentration were increased. Additionally, we identified that MiR-154-5p could bind to the 3'-UTR of POSTN and negative regulate POSTN. Knockdown of miR-154-5p increased osteogenic differentiation of BMMSCs. However, the molecular mechanisms governing differentiation of BMMSCs between Treg-cellderived exosomes and miR-154-5p has not been investigated.

There is accumulating evidence that microRNAs (miRNAs), long non-coding RNAs (IncRNAs), and circular RNAs (circRNAs) are closely related to the occurrence and development of various diseases, including inflammatory diseases, metabolic diseases, and cancer [57-59]. Long non-coding RNA (IncRNA) is an RNA molecule greater than 200 nucleotides in length and recent studies have also shown that IncRNAs play an important role in the osteogenic differentiation of BMMSCs[60-62]. Growing research has demonstrated that IncRNA modulates gene expression at multiple levels such as epigenetic, transcriptional and post transcriptional [63]. And IncRNAs could act as competitive endogenous RNAs and regulate the expression and activity of miRNAs. Among LncRNAs, $\mathrm{H} 19$ has been addressed in various cancers as an oncogene, and regulated cell proliferation, apoptosis and migration [64]. It has been found that $\mathrm{H} 19$ down-regulation modulated osteogenic differentiation of BMSCs from ovariectomized mouse, which suggested an important role of H19 in postmenopausal osteoporosis [65]. LncRNA H19 also mediates mechanical tension-induced osteogenesis of BMMSCs via FAK by sponging miR-138 [32]. Recent study showed that LncRNA H19 promotes odontoblastic differentiation of human dental pulp stem cells by regulating miR-140-5p and BMP-2/FGF9[66]. To our knowledge, no study has addressed the role of IncRNA H19 from Tregs-derived exosomes in osteogenic differentiation of BMMSCs. We therefore investigated the correlation between IncRNA H19 and miR-154-5p. In the present study, we observed IncRNA H19 from Tregs-derived exosomes could bind to miR-154-5p and negatively regulate its expression. And more importantly, our results indicated that IncRNA H19 could affect the osteogenic differentiation of BMMSCs in vitro by regulating POSTN expression via sponge miR-154-5p. These data indicated that $\mathrm{H} 19$ was involved in the regulation of BMMSCs through modulating miR-154-5p.

In conclusion, for the first time, our results indicated important role of exosomes from Tregs in regulating osteogenic differentiation of BMMSCs. It further revealed that IncRNA H19 from Tregs-derived exosomes plays a positive regulatory role in osteogenic differentiation of BMMSCs through miR-154-5p/POSTN axis. Our study exhibited the complicated regulation within ncRNA and provided a promising target to regulate the osteogenic potential of BMMSCs for treatment of periodontitis. Exosomes released from Tregs could be used as a new type of immunoregulation reagent for the treatment of periodontitis.

\section{Declarations}

\section{Authors' contributions}

Jing Zhang: Conception and design, Collection and/or assembly of data, Data analysis and interpretation, Manuscript writing; Zhigang Li: Collection and/or assembly of data; Data analysis and 
interpretation; Cuicui Liu: Collection and/or assembly of data; Yaxing Wu: Collection and/or assembly of data; Chenchen Li: Collection and/or assembly of data; Jian Meng: Provision of study material, Final approval of manuscript. The authors read and approved the final manuscript.

\section{Funding}

This work was supported by (31700814); Xuzhou Science and Technology Bureau project (KC18032). The funding bodies played no role in the design of the study and collection, analysis, and interpretation of data or writing of the manuscript.

\section{Availability of data and materials}

All data is available through the senior author.

\section{Ethics approval and consent to participate}

All animal procedures were approved by the Xuzhou Medical College's Ethics Committee.

\section{Consent for publication}

Not applicable.

\section{Competing interests}

Co-authors have no conflict of interest to report. Other authors declare no competing financial interests.

\section{Acknowledgements}

The authors would like to thank Professor Rui Li and Professor Xuekui Liu for technical advice. Jing Zhang gratefully acknowledge project support from the National Natural Science Foundation of China(Project Grant 31700814) and Xuzhou Science and Technology Bureau(Project Grant KC18032).

\section{References}

1. Pihlstrom BL, Michalowicz BS, Johnson NW. Periodontal diseases. Lancet. 2005;366(9499):18091820.

2. Su F, Liu SS, Ma JL, Wang DS, E LL, Liu HC. Enhancement of periodontal tissue regeneration by transplantation of osteoprotegerin-engineered periodontal ligament stem cells. Stem Cell Res Ther. 2015;6(1):22.

3. Guo S, Guo W, Ding Y, Gong J, Zou Q, Xie D, Chen Y, Wu Y, Tian W. Comparative study of human dental follicle cell sheets and periodontal ligament cell sheets for periodontal tissue regeneration. Cell Transplant. 2013; 22(6): 1061-1073.

4. Huang CY, Pelaez D, Dominguez-Bendala J, Garcia-Godoy F, Cheung HS. Plasticity of stem cells derived from adult periodontal ligament. Regen Med. 2009;4(6):809-821. 
5. Perez JR, Kouroupis D, Li DJ, Best TM, Kaplan L, Correa D. Tissue engineering and cell-based therapies for fractures and bone defects. Front Bioeng Biotechnol. 2018;6:105.

6. Liu Y, Zheng Y, Ding G, Fang D, Zhang C, Mark PB, Gronthos S, Shi S, Wang S. Periodontal ligament stem cell-mediated treatment for periodontitis in miniature swine. Stem Cells. 2008;26(4):1065-1073.

7. Chen FM, Gao LN, Tian BM, Zhang XY, Zhang YJ, Dong GY, Lu H, Chu Q, Xu J, Yu Y, Wu RX, Yin Y, Shi $\mathrm{S}$, JinY. Treatment of periodontal intrabony defects using autologous periodontal ligament stem cells: a randomized clinical trial. Stem Cell Res Ther. 2016;7:33.

8. Fakhry M, Hamade E, Badran B, Buchet R, Magne D. Molecular mechanisms of mesenchymal stem cell differentiation towards osteoblasts.World J Stem Cells. 2013; 5(4): 136-148.

9. Pilgram TK, Hildebolt CF, Dotson M, Cohen SC, Hauser JF, Kardaris E, Civitelli R. Relationships between clinical attachment level and spine and hip bone mineral density: data from healthy postmenopausal women. J Periodontol. 2002;73(3): 298-301.

10. 10. Chen $X$, Hu C, Wang G, Li L, Kong X, Ding Y, Jin Y. Nuclear factor- $K \beta$ modulates osteogenesis of periodontal ligament stem cells through competition with $\beta$-catenin signaling in inflammatory microenvironments. Cell Death Dis. 2013;4:e

11. Kim SH, Kim KH, Seo BM, Koo KT, Kim T, Seol YJ, Ku Y, Rhyu IC, Chung CP, Lee YM. Alveolar bone regeneration by transplantation of periodontal ligament stem cells and bone marrow stem cells in a canine peri-implant defect model: a pilot study. J Periodontol. 2009;80(11):1815-1823.

12. Zhang J, Li ZG, Si YM, Chen B, Meng J. The difference on the osteogenic differentiation between periodontal ligament stem cells and bone marrow mesenchymal stem cells under inflammatory microenviroments. Differentiation. 2014; 88(4-5): 97-105.

13. Shi Y, Wang Y, Li Q, Liu K, Hou J, Shao C, Wang Y. Immunoregulatory mechanisms of mesenchymal stem and stromal cells in inflammatory diseases. Nat Rev Nephrol. 2018;14(8):493-507.

14. Li N, Hua J. Interactions between mesenchymal stem cells and the immune system. Cell Mol Life Sci. 2017;74(13):2345-2360.

15. Wang S, Zhu R, Li H, Li J, Han Q, Zhao RC. Mesenchymal stem cells and immune disorders: from basic science to clinical transition. Front Med. $2019 ; 13(2): 138-151$.

16. Han Y, Li X, Zhang Y, Han Y, Chang F, Ding J. Mesenchymal Stem Cells for Regenerative Medicine. Cells. 2019;8(8):886.

17. Cardoso CR, Garlet GP, Crippa GE, Rosa AL, Júnior WM, Rossi MA, Silva JS. Evidence of the presence of T helper type 17 cells in chronic lesions of human periodontal disease. Oral Microbiol Immunol. 2009;24(1): 1-6.

18. Josefowicz SZ, Lu LF, Rudensky AY. Regulatory T cells:mechanisms of differentiation and function. Annu Rev Immunol. 2012;30:531-564.

19. Sakaguchi S. Regulatory T cells: key controllers of immunologic self-tolerance. Cell. 2000; 101(5): 455-458.

20. Shevach EM. Regulatory T cells in autoimmmunity. Annu Rev Immunol. 2000; 18: 423-449. 
21. Melief SM, Schrama E, Brugman MH, Tiemessen MM, Hoogduijn MJ, Fibbe WE, Roelofs $H$. Multipotent stromal cells induce human regulatory $\mathrm{T}$ cells through a novel pathway involving skewing of monocytes toward anti-inflammatory macrophages. Stem Cells. 2013; 31(9):1980-1991.

22. Carty F, Mahon BP, English K. The influence of macrophages on mesenchymal stromal cell therapy: passive or aggressive agents?. Clin Exp Immunol. 2017; 188(1): 1-11.

23. Kavanagh $\mathrm{H}$, Mahon BP. Allogeneic mesenchymal stem cells prevent allergic airway inflammation by inducing murine regulatory T cells. Allergy. 2011; 66(4):523-531.

24. Burr SP, Dazzi F, Garden OA. Mesenchymal stromal cells and regulatory T cells: the Yin and Yang of peripheral tolerance?. Immunol Cell Biol. 2013; 91(1):8-12.

25. Zaiss MM, Sarter K, Hess A, Engelke K, Böhm C, Nimmerjahn F, Voll R, Schett G, David JP. Increased bone density and resistance to ovariectomy-induced bone loss in FoxP3-transgenic mice based on impaired osteoclast differentiation. Arthritis Rheum. 2010; 62(8):2328-2338.

26. Liu Y, Wang L, Kikuiri T et al. Mesenchymal stem cell-based tissue regeneration is governed by recipient T lymphocytes via IFN-gamma and TNF-alpha. Nat Med. 2011; 17:1594-1601.

27. Jia B, Wang Z, Sun X, Chen J, Zhao J, Qiu X. Long noncoding RNA LINC00707 sponges miR-370-3p to promote osteogenesis of human bone marrow-derived mesenchymal stem cells through upregulating WNT2B. Stem Cell Res Ther. 2019;10(1): 67.

28. Gao Y, Xiao F, Wang C, Wang C, Cui P, Zhang X, Chen X. Long noncoding RNA MALAT1 promotes osterix expression to regulate osteogenic differentiation by targeting miRNA-143 in human bone marrow-derived mesenchymal stem cells. J Cell Biochem. 2018;119(8):6986-6996.

29. Tang Y, Liu L, Wang P, Chen D, Wu Z, Tang C. Periostin promotes migration and osteogenic differentiation of human periodontal ligament mesenchymal stem cells via the Jun amino-terminal kinases (JNK) pathway under inflammatory conditions. Cell Prolif. 2017;50(6):e12369.

30. Wang L, Wu F, Song Y, Li X, Wu Q, Duan Y, Jin Z. Long noncoding RNA related to periodontitis interacts with miR-182 to upregulate osteogenic differentiation in periodontal mesenchymal stem cells of periodontitis patients. Cell Death Dis. 2016;7(8):e2327.

31. Zhang F, Rong Z, Wang Z, Zhang Z, Sun D, Dong S, Xu J, Dai F. Periostin promotes ectopic osteogenesis of CTLA4-modified bone marrow mesenchymal stem cells. Cell Tissue Res. 2017;370(1): 143-151.

32. Wu J, Zhao J, Sun L, Pan Y, Wang H, Zhang W. Long non-coding RNA H19 mediates mechanical tension-induced osteogenesis of bone marrow mesenchymal stem cells via FAK by sponging miR138. Bone. 2018;108:62-70.

33. Azevedo RI, Minskaia E, Fernandes-Platzgummer A, Vieira AIS, da Silva CL, Cabral JMS, Lacerda JF. Mesenchymalstromal cells induce regulatory $T$ cells via epigenetic conversion of human conventional CD4 T cells in vitro. Stem Cells. 2020;38(8):1007-1019.

34. Luz-Crawford P, Kurte M, Bravo-Alegría J, Contreras R, Nova-Lamperti E, Tejedor G, Noël D, Jorgensen C, Figueroa F, Djouad F, Carrión F. Mesenchymal stem cells generate a CD4+CD25+Foxp3+ 
regulatory $T$ cell population during the differentiation process of Th1 and Th17 cells. Stem Cell Res Ther. 2013;4(3):65.

35. Khosravi M, Bidmeshkipour A, Moravej A, Hojjat-Assari S, Naserian S, Karimi MH. Induction of CD4(+)CD25(+)Foxp3(+) regulatory $T$ cells by mesenchymal stem cells is associated with RUNX complex factors. Immunol Res. 2018;66(1):207-218.

36. Gazdic M, Markovic BS, Arsenijevic A, Jovicic N, Acovic A, Harrell CR, Fellabaum C, Djonov V, Arsenijevic N, Lukic ML, Volarevic V. Crosstalk between mesenchymal stem cells and $T$ regulatory cells is crucially important for the attenuation of acute liver injury. Liver Transpl. 2018 ;24(5):687702.

37. Hong JW, Lim JH, Chung CJ, Kang TJ, Kim TY, Kim YS, Roh TS, Lew DH. Immune Tolerance of Human Dental Pulp-Derived Mesenchymal Stem Cells Mediated by CD4+CD25+FoxP3+ Regulatory TCells and Induced by TGF-beta1 and IL-10. Yonsei Med J. 2017;58(5):1031-1039.

38. Kreijveld E, Koenen HJPM, Hilbrands LB, Joosten I. Ex vivo expansion of human CD4+CD25high regulatory $\mathrm{T}$ cell from transplant recipients permits functional analysis of small blood samples. $\mathrm{J}$ Immunol Methods. 2006;314(1-2):103-113.

39. Chaput N, Taïeb J, Schartz NEC, André F, Angevin E, Zitvogel L. Exosome-based immunotherapy. Cancer Immunol Immunother. 2004;53(3):234-239.

40. Chaput N, Schartz NEC, André F, Taieb J, Novault S, Bonnaventure P, Aubert N, Bernard J, Lemonnier F, Merad M. Exosomes as potent cell-free peptide-based vaccine. II. Exosomes in CpG adjuvants effificiently prime naive Tc1 lymphocytes leading to tumor rejection. J. Immunol. 2004;172(4):21372146.

41. Chen L, Huang H, Zhang W, Ding F, Fan Z, Zeng Z. Exosomes Derived From T Regulatory Cells Suppress CD8+ Cytotoxic T Lymphocyte Proliferation and Prolong Liver Allograft Survival. Med Sci Monit. 2019;25:4877-4884.

42. Théry $C$, Duban $L$, Segura $E$, Véron $P$, Lantz $O$, Amigorena $S$. Indirect activation of naive CD $4+T$ cells by dendritic cell-derived exosomes. Nat Immunol. 2002; 3(12):1156-1162.

43. Segura E, Amigorena $S$, Thery $C$. Mature dendritic cells secrete exosomes with strong ability to induce antigen-specifific effector immune responses. Blood Cells Mol Dis 2005; 35(2):89-93.

44. Zhang H, Xie Y, Li W, Chibbar R, Xiong S, Xiang J. CD4+ T cell-released exosomes inhibit CD8+ cytotoxic T-lymphocyte responses and antitumor immunity. Cell and Mol Immunol. 2011; 8(1):23-30.

45. 4 Xie Y, Zhang H, Li W, Deng Y, Munegowda MA, Chibbar R, Qureshi M, Xiang J. Dendritic cells recruit T cell exosomes via exosomal LFA-1 leading to inhibition of CD8+ CTL responses through downregulation of peptide/MHC class I and Fas ligandmediated cytotoxicity. J Immunol. 2010;185(9):5268-5278.

46. 4 Busch A, Quast T, Keller S, Kolanus W, Knolle P, Altevogt P, Limmer A. Transfer of T cell surface molecules to dendritic cells upon CD4+ T cell priming involves two distinct mechanisms. J Immunol. 2008;181(6):3965-3973. 
47. 4 Mittelbrunn M, Gutiérrez-Vázquez C, Villarroya-Beltri C, González S, Sánchez-Cabo F, González MA, Bernad A, Sánchez-Madrid F. Unidirectional transfer of microRNA-loaded exosomes from T cells to antigen-presenting cells. Nat Commun. 2011;2:282.

48. Xiong YY, Gong ZT, Tang RJ, Yang YJ. The pivotal roles of exosomes derived from endogenous immune cells and exogenous stem cells in myocardial repair after acute myocardial infarction. Theranostics. 2021;11(3):1046-1058.

49. Hao Hu, Jiawei Wu, Cheng Cao, Likun Ma. Exosomes derived from regulatory $\mathrm{T}$ cells ameliorate acute myocardial infarction by promoting macrophage M2 polarization. IUBMB Life. 2020;72(11):2409-2419.

50. Yoshiba N, Yoshiba K, Hosoya A, Saito M, Yokoi T, Okiji T, Amizuka N, Ozawa H. Association of TIMP2 with extracellular matrix exposed to mechanical stress and its co-distribution with periostin during mouse mandible development. Cell Tissue Res. 2007;330(1):133-145.

51. Merle B, Garnero P. The multiple facets of periostin in bone metabolism. Osteoporos Int. 2012;23(4):1199-1212.

52. Bonnet N, Gineyts E, Ammann P, Conway SJ, Garnero P, Ferrari S. Periostin deficiency increases bone damage and impairs injury response to fatigue loading in adult mice. PLoS One. 2013;8(10):e78347.

53. Zhang F, Luo K, Rong Z, Wang Z, Luo F, Zhang Z, Sun D, Dong S, Xu J, Dai F. Periostin Upregulates Wnt/beta-Catenin Signaling to Promote the Osteogenesis of CTLA4-Modified Human Bone MarrowMesenchymal Stem Cells. Sci Rep. 2017;7:41634.

54. Jie Chen, Chengxian Ma, Yufeng Zhang, Shuai Pei, Mingyu Du, Yujie Zhang, Luxi Qian, Jianlin Wang , Li Yin , Xia He. MiR-154-5p Suppresses Cell Invasion and Migration Through Inhibiting KIF14 in Nasopharyngeal Carcinoma. Onco Targets Ther. 2020;13:2235-2246.

55. Xiuyu Wang, Shupeng Sun, Xiaoguang Tong, Quanfeng Ma, Hui Di, Tao Fu , Zhen Sun, Ying Cai, Weijia Fan, Qiaoli Wu , Yidi Li , Qiong Wang, Jinhuan Wang. MiRNA-154-5p inhibits cell proliferation and metastasis by targeting PIWIL1 in glioblastoma. Brain Res. 2017;1676:69-76.

56. Jianwei Li, Chen Hu, Lu Han, Lei Liu, Wei Jing, Wei Tang, Weidong Tian, Jie Long. MiR-154-5p regulates osteogenic differentiation of adipose-derived mesenchymal stem cells under tensile stress through the Wnt/PCP pathway by targeting Wnt11. Bone. 2015;78:130-41.

57. Cao J. The functional role of long non-coding RNAs and epigenetics. Biological Procedures Online. 2014;16, 11. doi: 10.1186/1480-9222-16-11.

58. Lv H, Sun Y, Zhang Y. MiR-133 is involved in estrogen deficiency-induced osteoporosis through modulating osteogenic differentiation of mesenchymal stem cells. Med Sci Monit. 2015;21:15271534.

59. Evans JR, Feng FY, Chinnaiyan AM. The bright side of dark matter: IncRNAs in cancer. J Clin Invest. 2016;126(8):2775-2782.

60. Sun X, Jia B, Qiu X, Chu H, Zhang Z, Wang Z, Zhao J. Potential functions of long non-coding RNAs in the osteogenic differentiation of human bone marrow mesenchymal stem cells. Mol Med Rep. 2019;19(1):103-114. 
61. Wang J, Liu S, Shi J, Liu H, Li J, Zhao S, Yi Z. The Role of IncRNAs in Osteogenic Differentiation of Bone Marrow Mesenchymal Stem Cells. Curr Stem Cell Res Ther. 2020;15(3):243-249.

62. Huo S, Zhou Y, He X, Wan M, Du W, Xu X, Ye L, Zhou X, Zheng L. Insight into the Role of Long Noncoding RNAs During Osteogenesis in Mesenchymal Stem Cells. Curr Stem Cell Res Ther. 2018;13(1):52-59.

63. Li Z, Tan H, Zhao W, Xu Y, Zhang Z, Wang M, Zhou X. Integrative analysis of DNA methylation and gene expression profiles identifies MIR4435-2HG as an oncogenic IncRNA for glioma progression. Gene. 2019;715:144012.

64. Ye Y, Shen A, Liu A. Long non-coding RNA H19 and cancer: A competing endogenous RNA. Bull Cancer. 2019;106(12):1152-1159.

65. Ping Zhou, Ying Li, Ruolin Di, Yi Yang, Songyan Meng, Fangfang Song, Lan Ma. H19 and Foxc2 synergistically promotes osteogenic differentiation of BMSCs via Wnt- $\beta$-catenin pathway. J Cell Physiol. 2019;234(8):13799-13806.

66. Zhong J, Tu X, Kong Y, Guo L, Li B, Zhong W, Cheng Y, Jiang Y, Jiang Q. LncRNA H19 promotes odontoblastic differentiation of human dental pulp stem cells by regulating miR-140-5p and BMP2/FGF9. Stem Cell Res Ther. 2020;11(1):202.

\section{Figures}

A
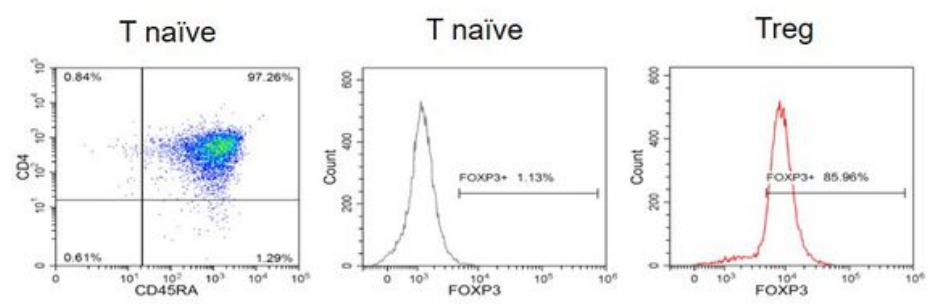

C

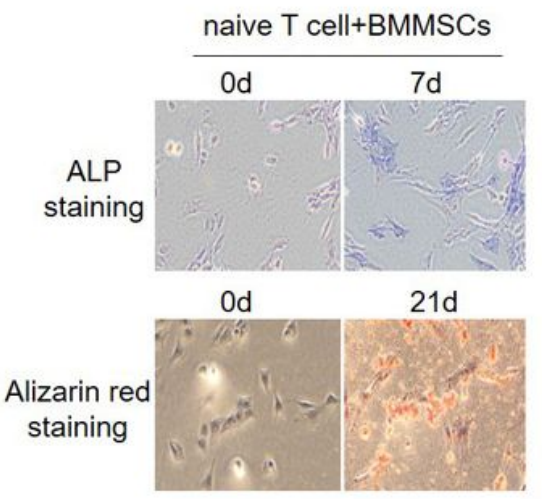

B

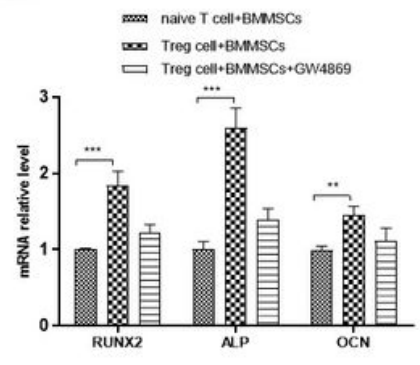

Treg cell+BMMSCs+GW4869

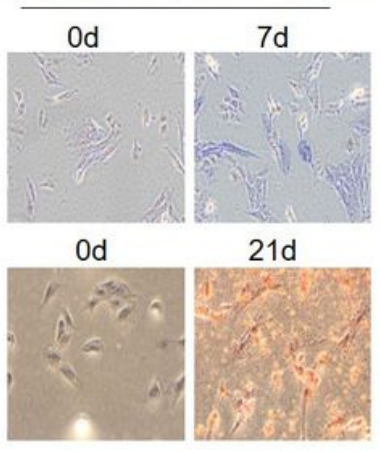

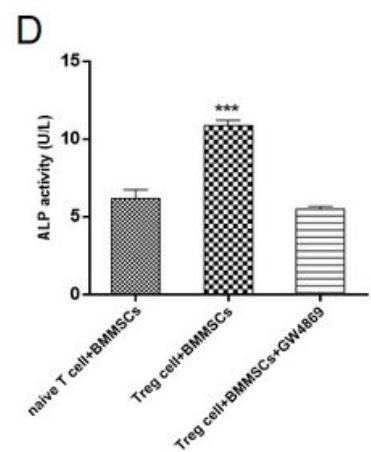

$\mathrm{E}$

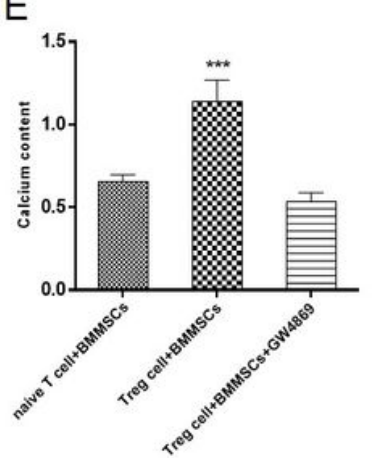

Figure 1 
Coculture of Tregs and BMMSCs can promote osteogenic differentiation of BMMSCs. (A) Isolation and identification of Naïve CD4+ T cells and Treg cells by flow cytometry. (B) The RUNX2, ALP and OCN mRNA expression levels of BMMSCs were determined by qRT-PCR when BMMSCs cocultured with Naïve CD4+ T cells, Tregs or Tregs+GW4869. The mRNA expression levels of RUNX2, ALP and OCN in Naïve CD4+ $T$ cells treatment group was arbitrarily set to 1, separately. (C)-(E) Osteogenic differentiations of BMMSCs were determined by ALP staining, Alizarin Red S, ALP activity assay and calcium content detection 7 or 21 days after osteogenic induction. Data are shown as mean \pm SEM $(n=3)$. Asterisks indicate significant differences from the control (*, $p<0.05 ; * \star, p<0.01 ; * * *, p<0.001)$.

A

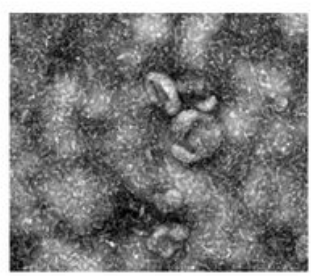

B

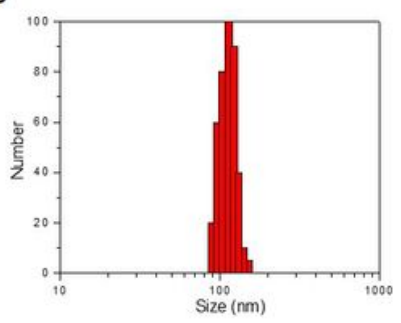

F

$\mathrm{E}$
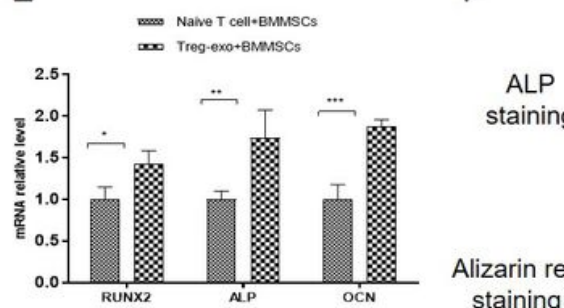

od

Od
C
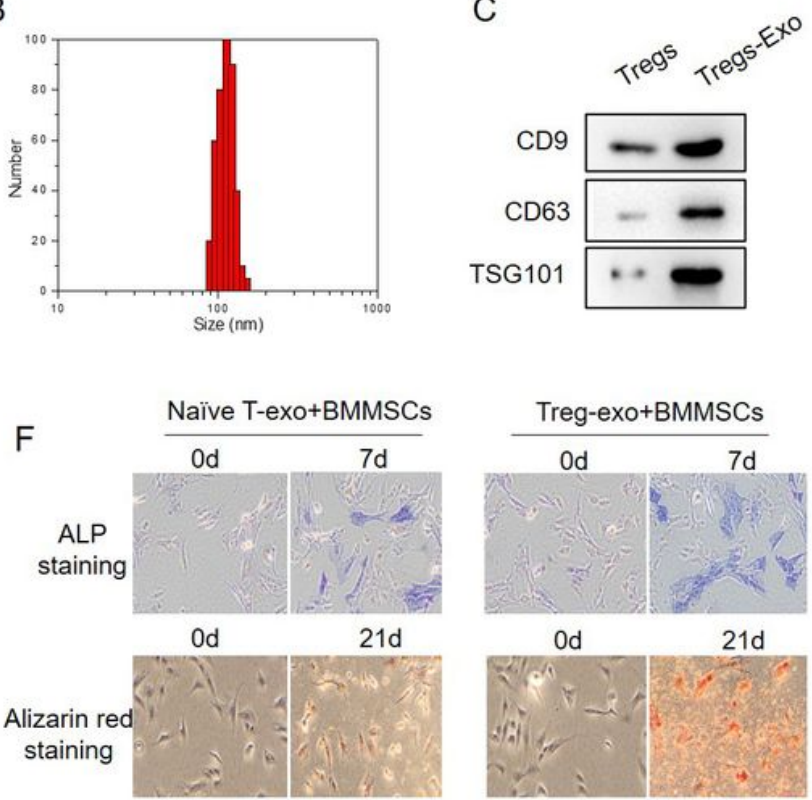
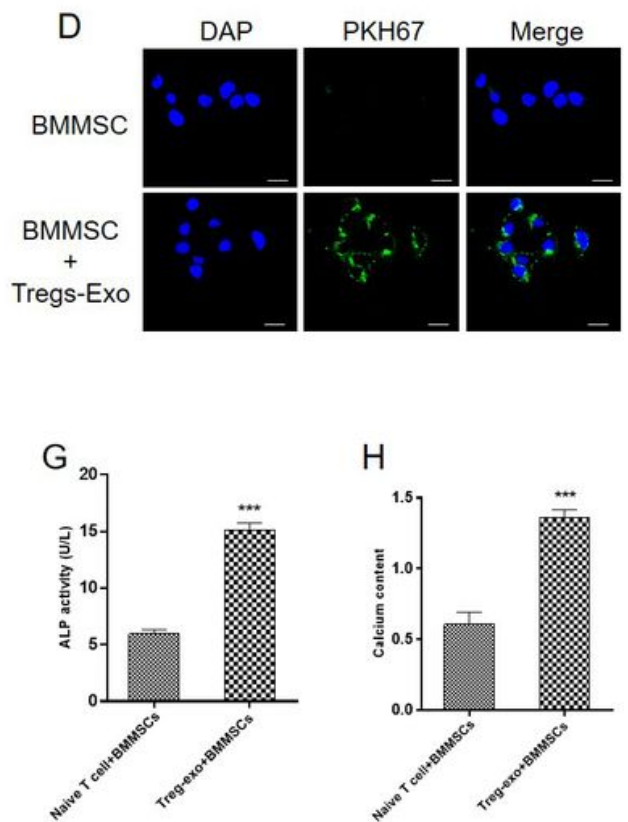

$\mathrm{H}$

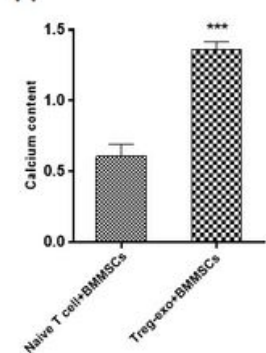

\section{Figure 2}

Coincubation of Tregs-derived exosomes and BMMSCs can promote osteogenic differentiation of BMMSCs. (A) The transmission electron microscope (TEM) image of exosomes isolated from Tregs, revealing the typical morphology and size (30 and $150 \mathrm{~nm}$ ). (scale bar, $200 \mathrm{~nm}$ ). (B) The particle diameter $(\mathrm{nm})$ of the population of small vesicles collected from Tregs was detected using qNano. (C) Western blot showed that exosome marker CD9, CD63 and TSG101 were expressed in exosomes. (D) The confocal image showing the internalization of PKH67-labeled exosomes by BMMSCs when added Tregs exosomes treatment. 4', 6-Diamidino-2-phenylindole was used to stain the nuclear of cells. (E) The RUNX2, ALP and OCN mRNA expression levels of BMMSCs were determined by qRT-PCR when BMMSCs co-cultured with Naïve CD4+ T cells or Tregs exosome. The mRNA expression levels of RUNX2, ALP and OCN in Naïve CD4+ $T$ cells treatment group was arbitrarily set to 1 , separately. $(F)-(H)$ Osteogenic differentiations of BMMSCs were determined by ALP staining, Alizarin Red S, ALP activity assay and calcium content detection 7 or 21 days after osteogenic induction when co-cultured with Naïve CD4+ T cells or Tregs exosome. Data are shown as mean \pm SEM $(n=3)$. Asterisks indicate significant differences from the control (*, p< 0.05; **, p< 0.01; ***, p< 0.001). 
A
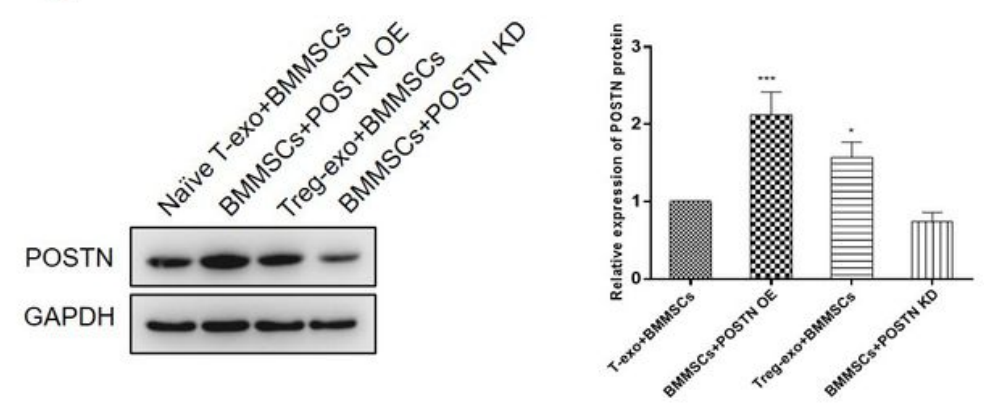

B
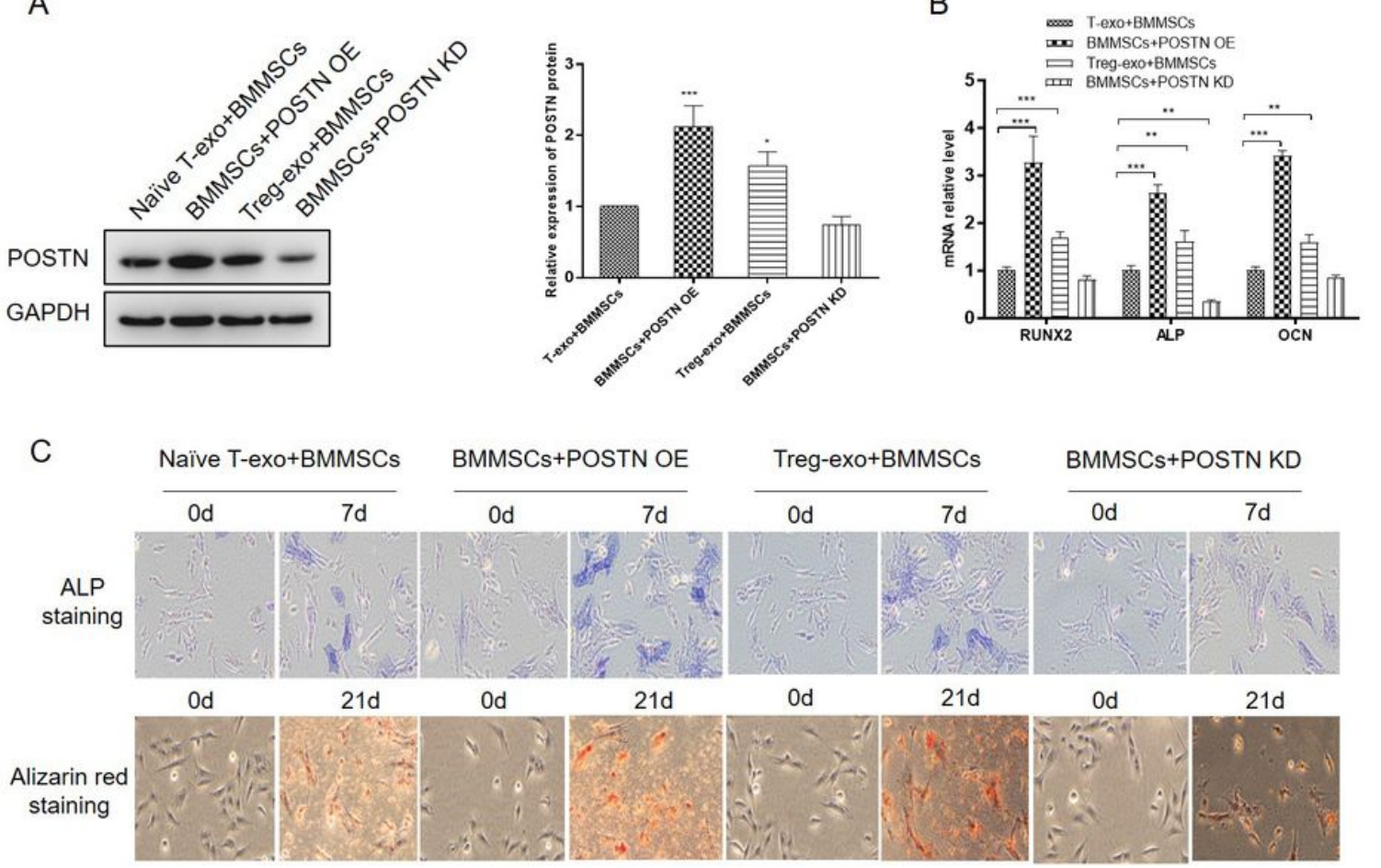

D

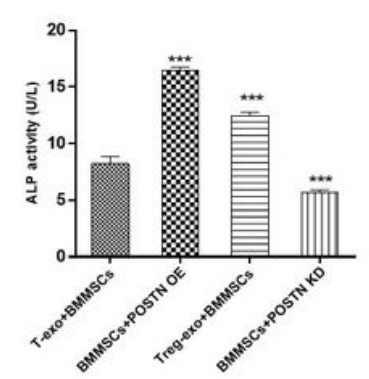

$E$

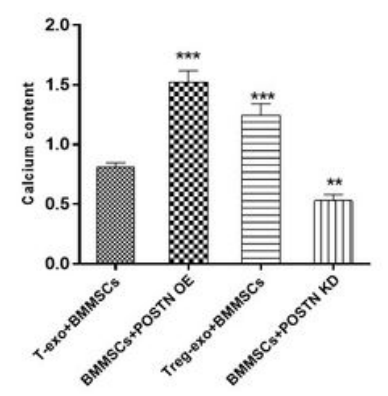

Figure 3

Tregs-derived exosomes promote the osteogenic differentiation of BMMSCs by regulating the expression of POSTN protein. (A) The expression of POSTN in BMMSCs by Western blot when BMMSCs co-cultured with Naïve CD4+ T cells, BMMSCs/POSTN OE, exosomes and BMMSCs/POSTN KD, separately. (B) The RUNX2, ALP and OCN mRNA expression levels of BMMSCs were determined by qRT-PCR when BMMSCs co-cultured with Naïve CD4+ T cells, BMMSCs/POSTN OE, exosomes and BMMSCs/POSTN KD . The mRNA expression levels of RUNX2, ALP and OCN in Naïve CD4+ T cells treatment group was arbitrarily set to 1, separately. (C)-(E) Osteogenic differentiations of BMMSCs were determined by ALP staining, Alizarin Red S, ALP activity assay and calcium content detection 7 or 21 days after osteogenic induction when co-cultured with Naïve CD4+ T cells, BMMSCs/POSTN OE, Treg-exosomes and BMMSCs/POSTN KD. Data are shown as mean \pm SEM $(n=3)$. Asterisks indicate significant differences from the control $(*, p$ $<0.05 ; * \star, p<0.01 ; * \star \star, p<0.001)$. 
A
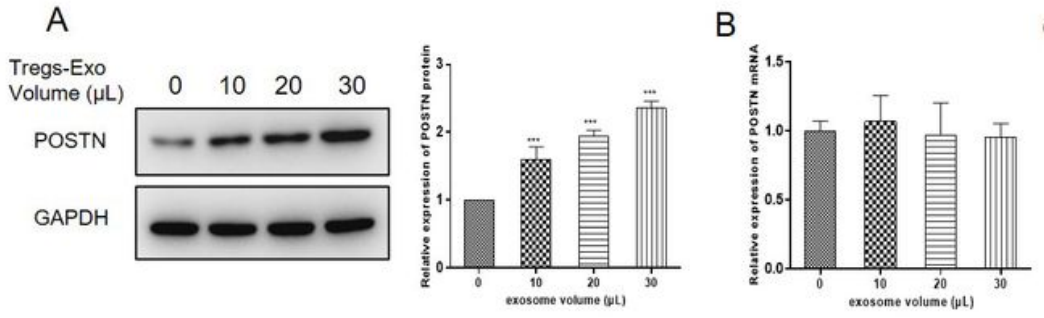

C

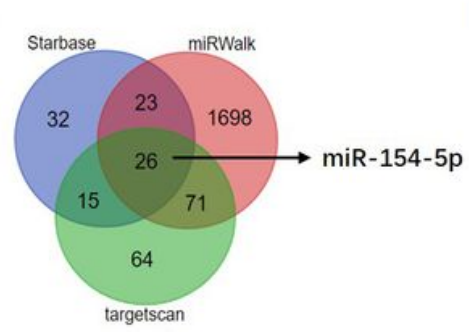

$\mathrm{E}$

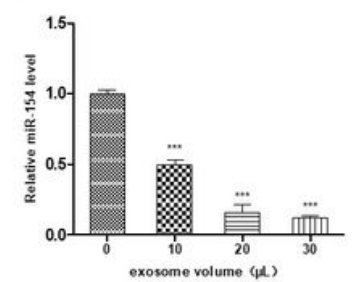

$\mathrm{F}$

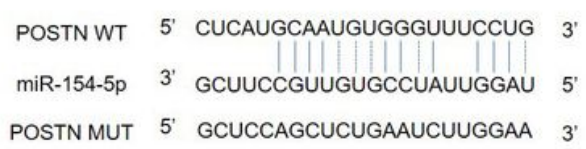

POSTN MUT 5' GCUCCAGCUCUgAaUCUUGgaA 3'

I

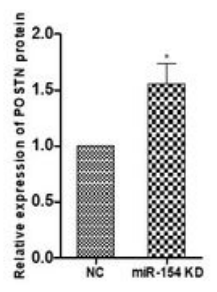

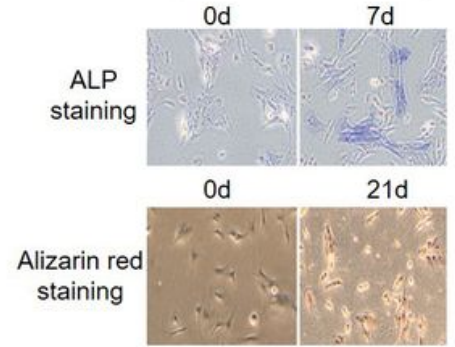

G
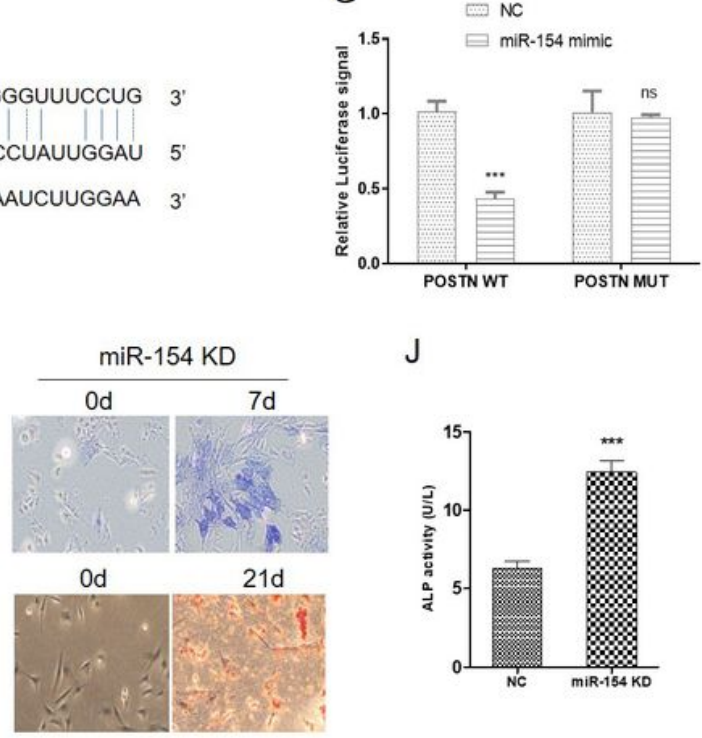

$J$

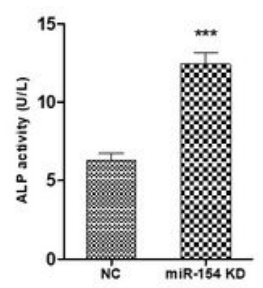

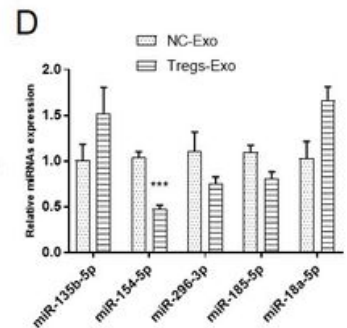

$\mathrm{H}$

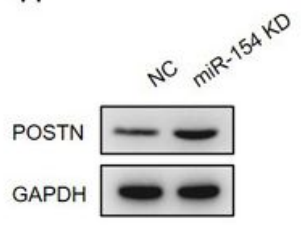

$\mathrm{K}$

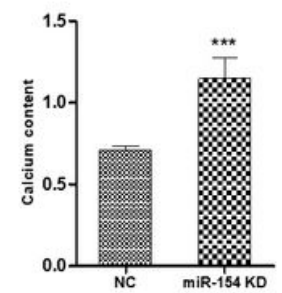

\section{Figure 4}

MiR-154-5p affects osteogenic differentiation of BMMSCs by regulating the expression of POSTN. (A) The protein expression of POSTN in BMMSCs by Western blot when BMMSCs co-cultured with different concentrations of exosomes. Concentration gradient: 0, 10, 20 and 30 $\mathrm{LL}$. (B) The mRNA expression of POSTN in BMMSCs by qRT-PCR when BMMSCs co-cultured with different concentrations of exosomes.

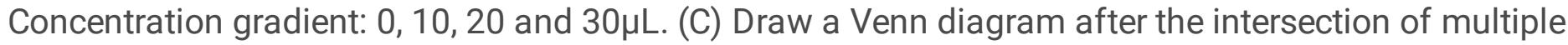
miRNAs databases. (D) The mRNA expression of the 5 screened miRNAs was detected by qRT-PCR when BMMSCs co-cultured with or without Tregs-derived exosomes. (E) The mRNA expression of miR-154-5p in BMMSCs by qRT-PCR when BMMSCs co-cultured with these concentrations of exosomes. (F) 3'-UTR base pairing diagram of miR-154-5p and POSTN. Replacement of Adenine bases with Uracil (A to $U$ ) can be used for the construction of mutant reporter. (G) Cells were co-transfected with miR-154-5p mimics and a luciferase reporter containing a fragment of the POSTN 3'-UTR harboring either the miR-154-5p binding site (POSTN-3'-UTR-WT) or a mutant (POSTN-3'-UTR-MUT). (H) The protein expression of POSTN in BMMSCs by Western blot when transfected with miR-154-5p inhibitor (KD). (I) -(K) Osteogenic differentiations of BMMSCs were determined by ALP staining, Alizarin Red S, ALP activity assay and calcium content detection 7 or 21 days after osteogenic induction when transfected with or without miR154-5p inhibitor $(K D)$. Data are shown as mean \pm SEM $(n=3)$. Asterisks indicate significant differences from the control $(*, p<0.05 ; * *, p<0.01 ; * \star *, p<0.001)$. 
A

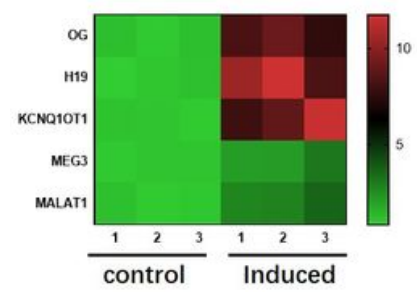

D
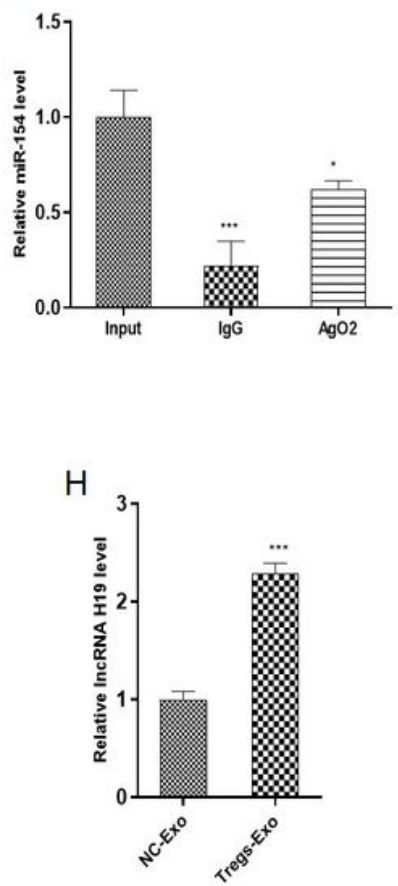

B

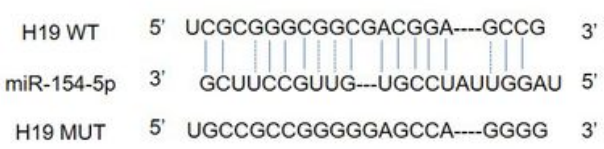

C

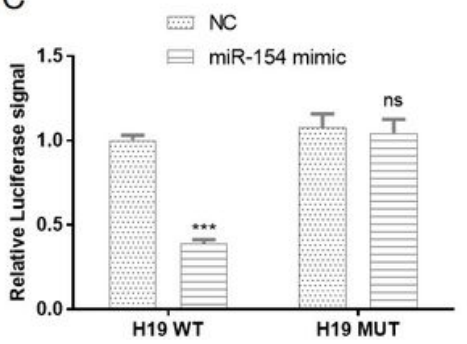

E

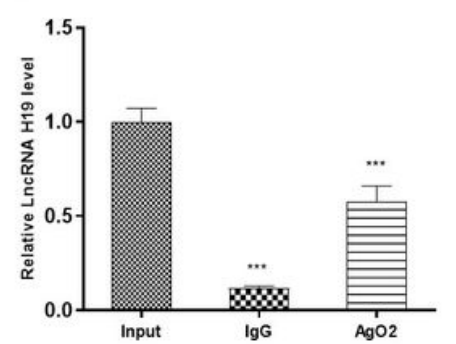

$\mathrm{F}$

AGO2

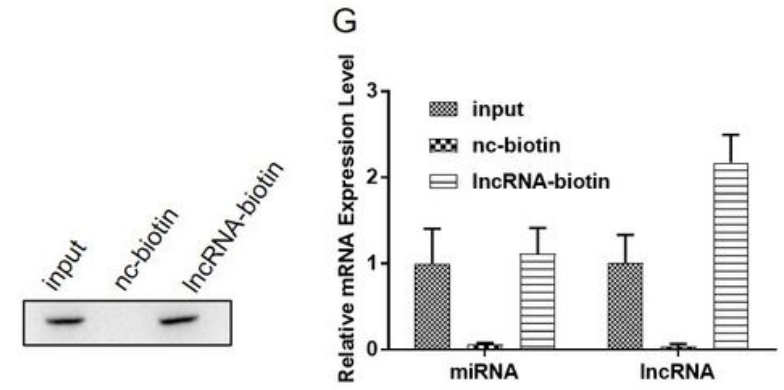

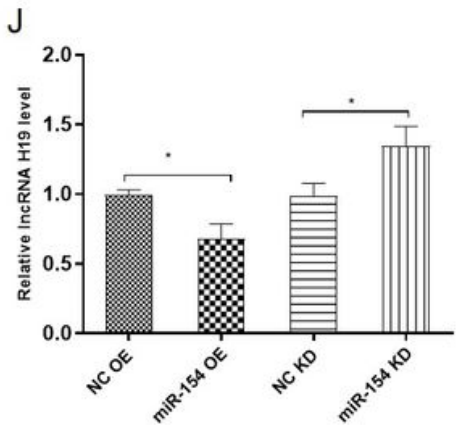

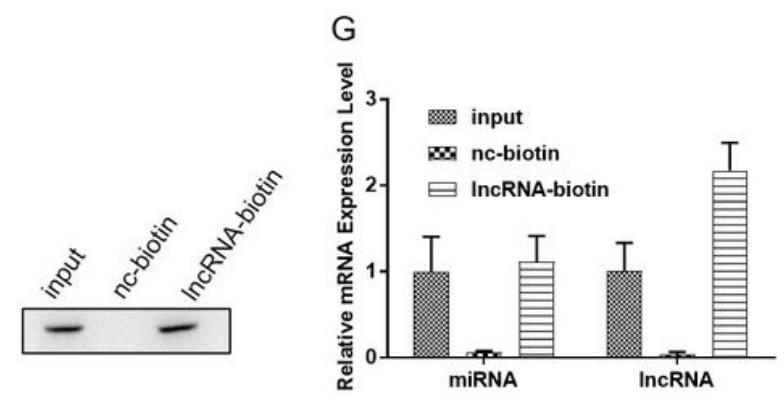

\section{Figure 5}

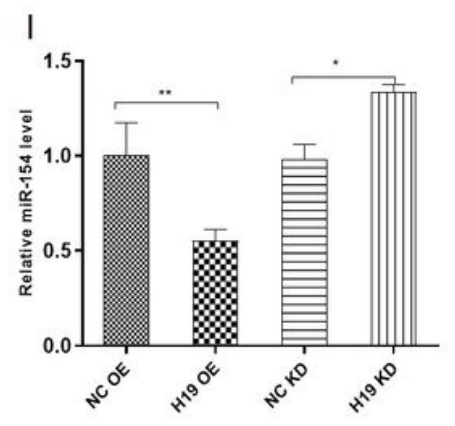

The highly expressed LncRNA H19 in Tregs can target miR-154-5p. (A) The mRNA expression of the selected IncRNAs was detected by qRT-PCR, and a heat map was drawn. (B) 3'-UTR base pairing diagram of miR-154-5p and H19. Replacement of Cytosine bases with Guanine ( $C$ to $G$ ) can be used for the construction of mutant reporter. (C) Cells were co-transfected with miR-154-5p mimics and a luciferase reporter containing a fragment of the H19 3'-UTR harboring either the miR-154-5p binding site ( $\mathrm{H} 19-3$ 'UTR-WT) or a mutant (H19 -3'-UTR-MUT). (D)-(E) RNA immunoprecipitation with an anti-Ago2 antibody was used to assess endogenous Ago2 binding to RNA in BMMSCs, IgG was used as the control. H19 and miR-154-5p levels were determined by qRT-PCR and presented as fold enrichment in Ago2 relative to input. (F)-(G) RNA pull-down assays were used to examine the interaction of $\mathrm{H} 19$ and Ago2 in BMMSCs. H19 and miR-154-5p levels were determined by qRT-PCR. (H) The mRNA expression of H19 in BMMSCs by qRT-PCR when BMMSCs co-cultured with conventional T cells (NC) exosomes or Tregs exosomes. (I) The mRNA expression of miR-154-5p in BMMSCs by qRT-PCR in NC OE, H19 OE, NC KD and H19 KD BMMSCs cell lines. (J) The mRNA expression of H19 in BMMSCs by qRT-PCR in NC OE, miR-154-5p OE, NC KD and miR-154-5p KD BMMSCs cell lines. All experiments were repeated three times. Relative 
expressions of IncRNA-H19 and miR-154-5p were normalized by $\beta$-actin and U6 in qRT-PCR, respectively. Data are shown as mean \pm SEM $(n=3)$. Asterisks indicate significant differences from the control $(*, p<$ $0.05 ; * *, p<0.01 ; * \star *, p<0.001)$.
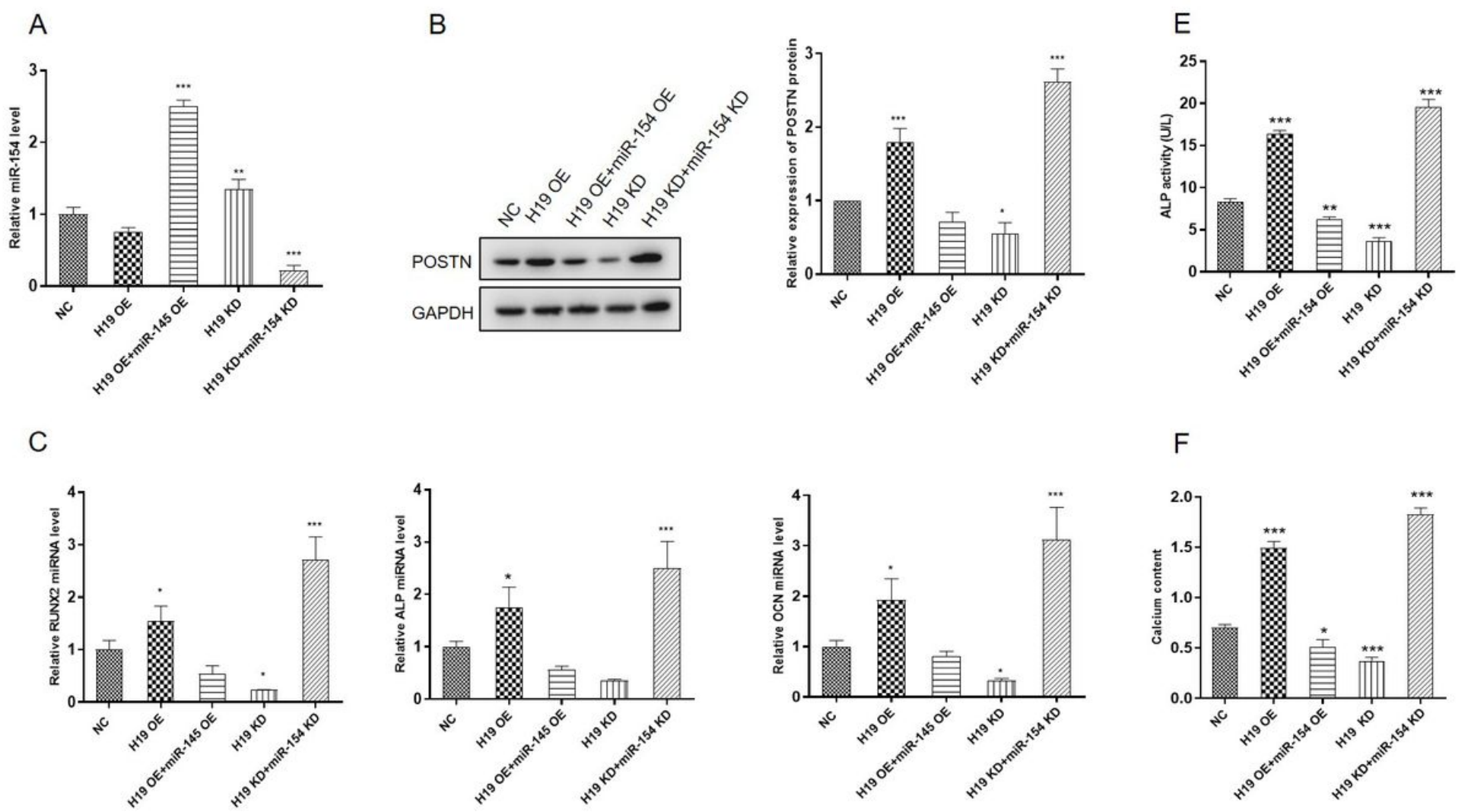

D
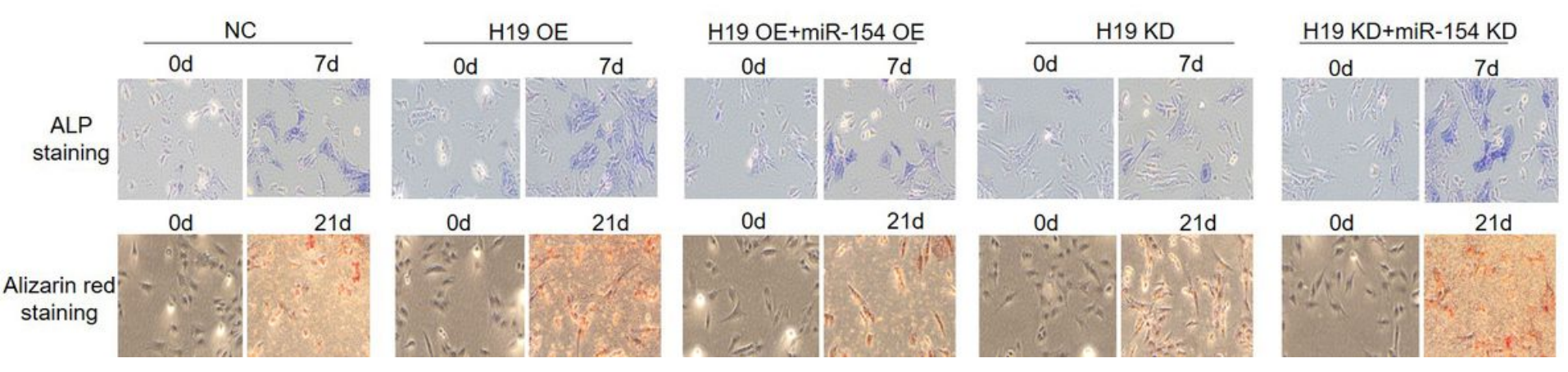

\section{Figure 6}

LncRNA H19 regulates POSTN expression and osteogenic differentiation of BMMSCs by inhibiting miR154-5p. (A) The mRNA expression of miR-154-5p in BMMSCs by qRT-PCR in NC, H19 OE, H19 OE + miR154-5p OE, H19 KD and H19 KD + miR-154-5p KD BMMSCs cell lines. (B) The protein expression of POSTN in BMMSCs by Western blot in NC, H19 OE, H19 OE + miR-154-5p OE, H19 KD and H19 KD + miR154-5p KD BMMSCs cell lines. (C) The RUNX2, ALP and OCN mRNA expression levels of BMMSCs were determined by qRT-PCR in NC, H19 OE, H19 OE + miR-154-5p OE, H19 KD and H19 KD + miR-154-5p KD BMMSCs cell lines. (D)-(F) Osteogenic differentiations of BMMSCs were determined by ALP staining, Alizarin Red S, ALP activity assay and calcium content detection 7 or 21 days after osteogenic induction 
in NC, H19 OE, H19 OE + miR-154-5p OE, H19 KD and H19 KD + miR-154-5p KD BMMSCs cell lines. Data are shown as mean \pm SEM $(n=3)$. Asterisks indicate significant differences from the control $(*, p<0.05$; $\star *, p<0.01 ; * \star *, p<0.001)$.

A

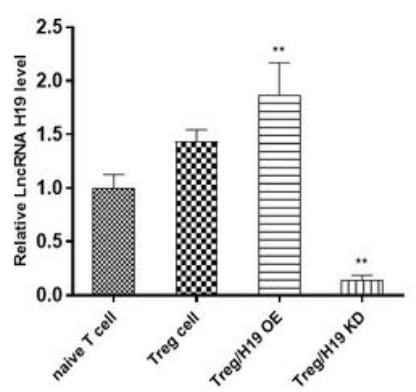

B

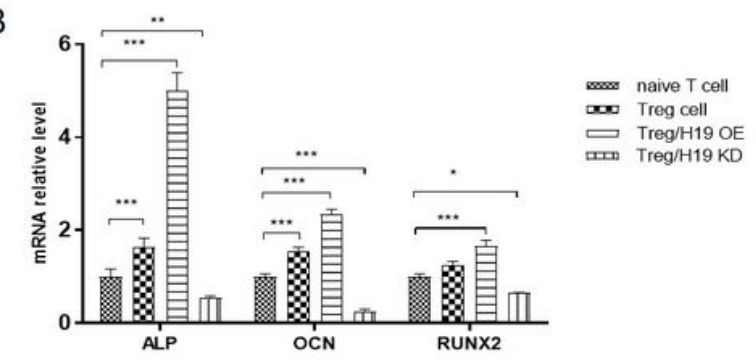

C
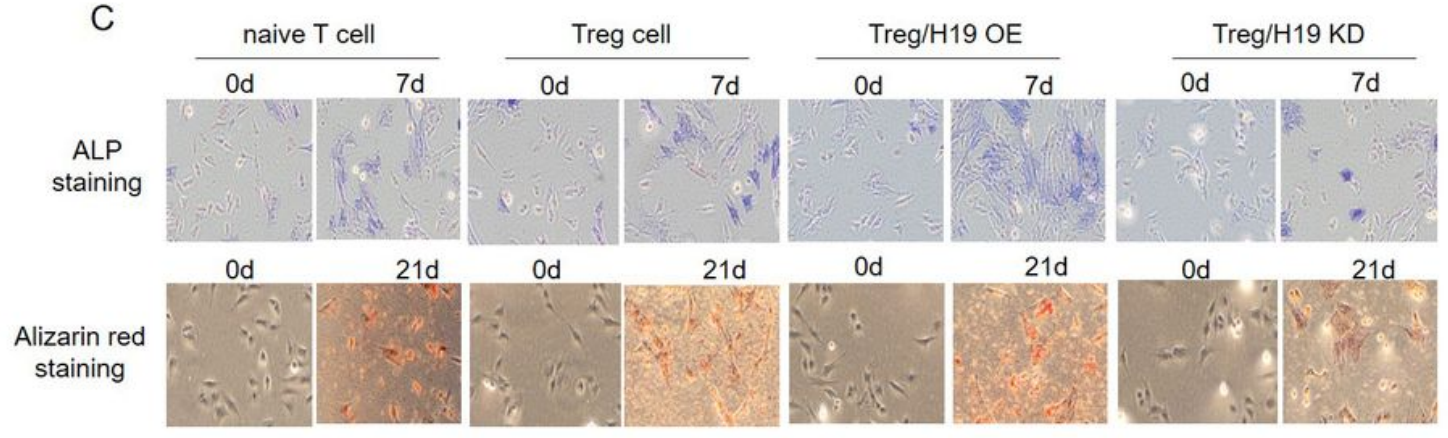

21d

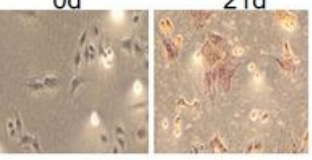

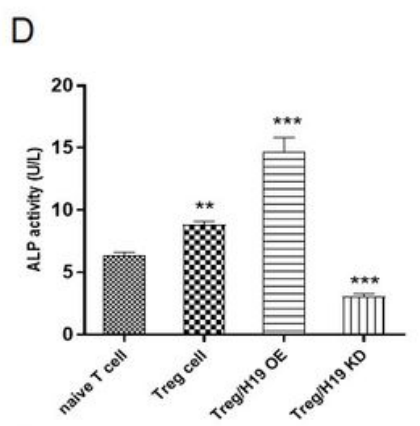

E

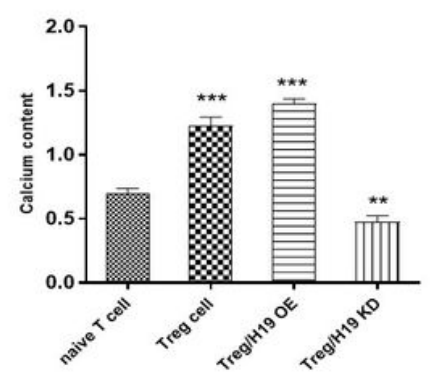

\section{Figure 7}

Tregs-derived exosomes LncRNA H19 participates in regulating osteogenic differentiation of BMMSCs in vivo. (A) The mRNA expression of H19 in BMMSCs by qRT-PCR when BMMSCs co-cultured with Naïve CD4+ T cells, Tregs, Treg/H19 OE and Treg/H19 KD. (B) The RUNX2, ALP and OCN mRNA expression levels of BMMSCs were determined by qRT-PCR when BMMSCs co-cultured with Naïve CD4+ T cells, Tregs, Treg/H19 OE and Treg/H19 KD. (C)-(E) Osteogenic differentiations of BMMSCs were determined by ALP staining, Alizarin Red S, ALP activity assay and calcium content detection 7 or 21 days after osteogenic induction when BMMSCs co-cultured with Naïve CD4+ T cells, Tregs, Treg/H19 OE and Treg/H19 KD. Data are shown as mean \pm SEM $(n=3)$. Asterisks indicate significant differences from the control (*, p<0.05; **, p<0.01; ***, p<0.001).

\section{Supplementary Files}

This is a list of supplementary files associated with this preprint. Click to download.

- Figures1.tif 